\title{
Design and Analysis of Ultra-Wideband Split Transmit Virtual Aperture Array for Through-the-Wall Imaging
}

\author{
Biying Lu, Yang Zhao, Xin Sun, and Zhimin Zhou \\ School of Electronic Science and Engineering, National University of Defense Technology, Changsha 410073, China \\ Correspondence should be addressed to Biying Lu; joelu76@aliyun.com
}

Received 15 February 2013; Accepted 29 May 2013

Academic Editor: Krzysztof Kulpa

Copyright (C) 2013 Biying Lu et al. This is an open access article distributed under the Creative Commons Attribution License, which permits unrestricted use, distribution, and reproduction in any medium, provided the original work is properly cited.

\begin{abstract}
The concept of virtual aperture and the point spread function for designing and characterizing ultra-wideband near-field multipleinput multiple-output active imaging array are investigated. Combining the approach of virtual aperture desynthesis with the monostatic-to-bistatic equivalence theorem, a kind of linear UWB MIMO array, the split transmit virtual aperture (STVA) array, was designed for through-the-wall imaging. Given the virtual aperture, the STVA array is the shortest in physical aperture length. The imaging performance of the designed STVA array in the near field is fully analyzed through both numerical and measured data. The designed STVA array has been successfully applied to imaging moving targets inside buildings.
\end{abstract}

\section{Introduction}

Through-the-wall imaging (TWI) is an emerging technology that has a variety of potential applications including earthquake rescue, covert target detection, surveillance, and reconnaissance. Ultra-wideband (UWB) microwave imaging has been identified as a viable approach for TWI. Typically, a synthetic aperture approach is employed for imaging purposes while fixed aperture (i.e., antenna array) rather than synthetic aperture imaging systems preferred in such hostile or time-sensitive applications [1-9].

To simplify the array imaging system, two kinds of arrays, the switched antenna array [1-5] and the multiple input multiple output (MIMO) array [6-9], have mostly been used. The switched array concept is based on one transmit array and one receive array. The transmit array switches between antenna elements one at a time. The receive array also switches between antenna elements one at a time. In this way, the number of required transmit and receive antennas is significantly saved. Moreover, using the high speed electronic switch, the time to acquire a data set across the aperture is greatly reduced compared to that of a synthetic aperture imaging system [1]. MIMO array imaging is characterized by using multiple transmit antennas to transmit orthogonal waveforms simultaneously and by using multiple receive antennas to receive the scattered waveforms from the target simultaneously. Two types of MIMO array, array with widely separated antenna elements and array with collocated transmit and receive antennas, have been proposed $[10,11]$. For array imaging, the latter is preferred, and the use of orthogonal waveform is to increase the update rate rather than using diversity to improve the detection performance.

For active microwave imaging, both the switched antenna array and the MIMO array exhibit similar advantages of fast data acquisition and require less antenna elements. Further, the properties of both arrays are usually analyzed using the equivalent one-way co-array [6-9, 12-14] or two-way virtual aperture array $[1-5,15]$. Considering we are concentrating on two-way active microwave imaging, the more versatile term of MIMO array and the concept of virtual aperture are used in the following section.

The configuration of MIMO array has great effects on radar imaging, such as resolution and peak side lobe level. The theory of virtual aperture (co-array) together with its beam pattern provides a unified and convenient method for analyzing and designing MIMO arrays under narrow band far-field condition $[1-7,12,16]$. 
The concept of virtual aperture (co-array) was extended to ultra wide band (UWB) imaging MIMO array in $[8,9,12-$ 14 ], with a modified definition of the beam pattern, which is a function of both the azimuth direction and the additional dimension of time or range [17-20]. UWB MIMO array design methods based on the concept of virtual aperture have been presented in $[8,9,14]$.

Unfortunately, the same virtual aperture may be synthesized from MIMO arrays with different configurations. Further, high cross-range resolution of the UWB MIMO array imaging system requires large accumulation angle of the array to the imaging location, which conflicts with the farfield condition assumption of the virtual array approach. And the radar cross section (RCS) of a point scatterer is dependent on the imaging geometry of the MIMO array. Taking all these factors into account, a UWB MIMO array with the minimal physical aperture among those synthesizing the same virtual aperture, the split transmit virtual aperture array (STVA), for through the wall imaging application is presented. The performance of the designed array is fully analyzed using the point spread function (PSF), and the MIMO array has been successfully applied to image targets inside buildings.

This paper is organized as follows. In Section 2, the concept of co-array and virtual aperture together with the radiation pattern for wideband MIMO array is explained. The PSF of UWB array applicable for both far-and nearfield conditions is presented in Section 3. The equivalence of PSF and beam pattern under far-field narrow band condition is also demonstrated. In Section 4, the linear UWB MIMO array design method is described, and a specific array configuration, the STVA, for TWI is proposed. The performance of the designed array is analyzed using the PSF. The experimental imaging results of the UWB MIMO imaging system are presented in Section 5. Conclusions are summarized in the final section.

\section{Co-Array and Virtual Aperture}

2.1. Co-Array. For each two-way array, an equivalent array can be synthesized whose one-way beam pattern is identical to a two-way pattern of the initial array. The aperture function of the synthesized array is referred to as effective aperture. The most famous effective array for MIMO array is the co-array.

Consider a linear two-way MIMO array composed of $M$ transmit and $N$ receive omnidirectional elements located along the $x$-axis as shown in Figure 1. The location of the $m$ th transmit element is $\mathbf{r}_{t, m}=\left(x_{t, m}, 0\right), m=0,1,2, \ldots, M-1$, and the location of the $n$th receive element is $\mathbf{r}_{r, n}=\left(x_{r, n}, 0\right)$, $n=0,1,2, \ldots, N-1$. For active imaging, the equivalent coarray consists of $M N$ one-way element and the location of the $i$ th synthesized element (virtual element) of the co-array is defined as [16]

$$
\mathbf{r}_{c, i}=\left(x_{c, i}, 0\right)=\mathbf{r}_{t, m}+\mathbf{r}_{r, n}=\left(x_{t, m}+x_{r, n}, 0\right)
$$

where $i=(m+1)(n+1)-1$ for $m=0,1,2, \ldots, M-1$ and $n=0,1,2, \ldots, N-1$.

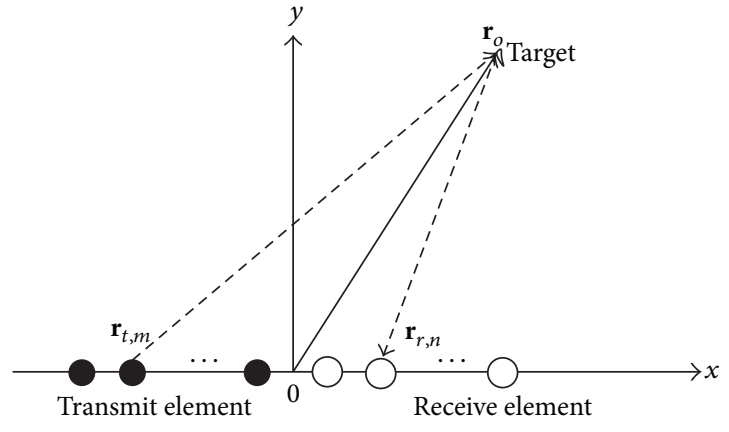

FIGURE 1: Linear transmit and receive arrays.

The weighting coefficient of the $i$ th virtual element of the co-array is

$$
w_{c, i}=w_{t, m} w_{r, n}
$$

where $w_{t, m}$ and $w_{r, n}$ are the weighting coefficients of the $m$ th transmit and $n$th receive elements of the MIMO array, respectively.

It should be noted that (1) and (2) are defined under the condition that no redundancy exists within the co-array. If different transmit/receive pairs within the MIMO array result in the same virtual element position, the corresponding weights at the same virtual element shall be summed.

Under narrow band far-field condition, assuming that the steering is fixed and pointed broadside, the performance of a one-way array may be fully described with the well-known beam pattern or radiation pattern, given by the Fourier transform of the array aperture weighting function (or in its discrete form). The radiation pattern of the two-way MIMO array is the beam pattern of the equivalent co-array, given as

$$
P_{\mathrm{MIMO}}(\theta)=P_{c}(\theta)=\sum_{i=0}^{M N-1} w_{c, i} e^{-j k x_{c, i} \sin \theta}
$$

where $\theta$ is the incidence angle from the normal of the aperture and $k=2 \pi / \lambda$ is the wave number with $\lambda$ as the wavelength of the signal. Substitute (1) and (2) into (3), $P_{\mathrm{MIMO}}(\theta)$ can be expressed as

$$
P_{\mathrm{MIMO}}(\theta)=\sum_{m=0}^{M-1} w_{t, m} e^{-j k x_{t, m} \sin \theta} \sum_{n=0}^{N-1} w_{r, n} e^{-j k x_{r, n} \sin \theta} .
$$

The first sum of (4) is the beam pattern of the transmit array $P_{t}(\theta)$, and the second sum is the beam pattern of the receive array $P_{r}(\theta)$. Thus, the radiation pattern of the two-way MIMO array is the multiplication of the transmit and receive patterns.

The concept of co-array was extended to wideband imaging MIMO array in $[8,9,12-14,17]$. It should be noted that the equivalent concept of effective array other than coarray was used in $[8,9,17]$. For narrow band far-field imaging, the co-array together with its radiation pattern can fully describe the overall performance over the azimuth direction $\theta$ of the MIMO array. However, for wideband far-field imaging, 
the radiated waveforms vary throughout time and angle, even in the far fields of the array. Therefore, the radiation pattern is a function of both the azimuth direction $\theta$ and the additional dimension of time or range. The wideband radiation pattern of the co-array is expressed as $[14,15]$

$$
P_{c}(\theta, t)=\sum_{i=0}^{M N-1} w_{c, i} A\left(t-\frac{x_{c, i} \sin \theta}{c}\right) e^{-j k x_{c, i} \sin \theta}
$$

where $k$ is the wavenumber of the central carrier frequency, $c$ is the speed of light, and $A(t)$ is the envelope of the transmitted wideband pulse, possibly after pulse compression. In the ideal monochromatic case, $A(t)$ is a constant equal to unity.

To compare with narrowband beam pattern, the time dimension of the wideband beam pattern must be reduced. There are many ways to reduce the time dimension of the wideband beam pattern. The commonly used method is to take the maximum time response at each angular location $[17,18]$

$$
P_{c}(\theta)=\max _{t}\left|P_{c}(\theta, t)\right|
$$

It should be noted that the method loses the information concerning the temporal shape of the radiation pattern.

2.2. Virtual Aperture. In the field of radar imaging, the concept of two-way virtual aperture rather than the coarray is usually adopted to analyze the MIMO array. The equivalent monostatic illumination angle is approximated by the bisector of the bistatic angle of the measurement using the physical-optic model for bistatic scattering of point scatterers at small bistatic angles [19]. Based on this theorem, an equivalent monostatic transmit/receive element (virtual element) located midway between the transmit and the receive elements was introduced under the far-field condition in [4]. Therefore, the location of the $i$ th virtual element of the virtual aperture for the MIMO array mentioned above is given as

$$
\mathbf{r}_{v, i}=\left(x_{v, i}, 0\right)=\left(\frac{x_{t, m}+x_{r, n}}{2}, 0\right)
$$

where $i=(m+1)(n+1)-1$ for $m=0,1,2, \ldots M-1$ and $n=0,1,2, \ldots, N-1$. The weighting coefficients of the virtual elements are the same as those of co-array elements, that is, $w_{v, i}=w_{c, i}$ for $i=0,1,2, \ldots, M N-1$.

Within the assumption that the wave field propagates with half of the actual speed, the monostatic transmit/receive element can be treated as one-way element at the same location. Thus, the radiation pattern of the virtual array for wideband far-field imaging is

$$
P_{v}(\theta)=\sum_{i=0}^{M N-1} w_{v, i} A\left(t-\frac{2 x_{v, i} \sin \theta}{c}\right) e^{-j 2 k x_{v, i} \sin \theta} .
$$

Note that $w_{v, i}=w_{c, i}$ and $x_{v, i}=x_{c, i} / 2$ for $i=0,1,2, \ldots, M N-$ 1 , we get

$$
P_{v}(\theta)=P_{c}(\theta)
$$

Equation (9) indicates the equivalence of the concept of the one-way co-array and the concept of the two-way virtual aperture. In this paper, we are mainly concerned about active imaging. The concept of the two-way virtual aperture is adopted.

\section{Point Spread Function}

Beam pattern mentioned above can fully describe the overall performance of the array in the far field. Unfortunately, it does not work in the near field. Actually, an imaging system can be fully characterized by the point spread function (PSF) defined as the response of the imaging system to an ideal point source, despite the variations of the transmitted waveform, angle, and distance of focus. The main lobe width of the PSF is a measurement of achievable resolution the grating lobes location and levels of the PSF determine the ambiguity region and their intensities, and the side lobe level of the PSF indicates the capabilities of distinguishing weaker scatterers in the proximity of the strong ones.

For narrow band far-field imaging, the equivalence between the virtual aperture and the original MIMO array has been demonstrated. Unfortunately, there are increasing approximate errors with shorter focus distance and wider band of transmitted signal. The approximation error for the wide band imaging MIMO array can be estimated and analyzed based on the analysis of the PSF of the virtual aperture and the PSF of the original MIMO array.

The imaging geometry of the MIMO array with an ideal point scatterer located at $\mathbf{r}_{o}=\left(x_{o}, y_{o}\right)$ is shown in Figure 1. Suppose $S(\omega)$ is the spectrum of the transmitted wideband signal $s(t)$, then $s(t)$ is the inverse Fourier transform of $S(\omega)$, given by

$$
s(t)=\frac{1}{2 \pi} \int_{-\infty}^{\infty} S(\omega) e^{j \omega t} d \omega
$$

Ideally, if the signal $s(t)$ is time limited, the spectrum $S(\omega)$ spreads all over the frequency and vice versa. Practically, the transmitted waveform is specially designed such that it is approximately both time limited and bandwidth limited. Further, the spectrum $S(\omega)$ is approximated by its uniformly sampled discrete values, resulting in the well-known stepped frequency (SF) waveform. Suppose the frequency bandwidth of the spectrum is $\left[f_{0}, f_{P-1}\right]$, a stepped frequency approximation to (10) is given as

$$
\begin{gathered}
s(t)=\sum_{p=0}^{P-1} S\left(f_{p}\right) e^{j 2 \pi f_{p} t}, \\
f_{p}=\left(f_{0}+p \Delta f\right), \quad p=0,1, \ldots, P-1,
\end{gathered}
$$

where $S\left(f_{p}\right)$ can be considered as the frequency weighting function of the SF waveform. For simplicity, we use unity weighting function with $S\left(f_{p}\right)=1$ in the following text. It should be noted that the $P$ frequency samples are usually transmitted in the form of gated narrowband pulse, step by step. Further description of the SF waveform can be found in $[5,13]$. 
Under the assumption of free space propagation and for the $p$ th frequency, the two-way Green's function of the $m$ th transmit and $n$th receive pair to the point scatterer is given by

$$
\begin{aligned}
G & \left(\mathbf{r}_{t, m}, \mathbf{r}_{r, n}, \mathbf{r}_{o}, f_{p}\right) \\
& =\frac{1}{4 \pi\left|\mathbf{r}_{t, m}-\mathbf{r}_{o}\right|} e^{-j k_{p}\left|\mathbf{r}_{t, m}-\mathbf{r}_{o}\right|} \cdot\left(\frac{1}{4 \pi\left|\mathbf{r}_{r, n}-\mathbf{r}_{o}\right|} e^{-j k_{p}\left|\mathbf{r}_{r, n}-\mathbf{r}_{o}\right|}\right) \\
& =\frac{1}{16 \pi^{2}\left|\mathbf{r}_{t, m}-\mathbf{r}_{o}\right|\left|\mathbf{r}_{r, n}-\mathbf{r}_{o}\right|} e^{-j k_{p}\left(\left|\mathbf{r}_{t, m}-\mathbf{r}_{o}\right|+\left|\mathbf{r}_{r, n}-\mathbf{r}_{o}\right|\right)}
\end{aligned}
$$

where $k_{p}=2 \pi / \lambda_{p}$ is the wavenumber and $\lambda_{p}=c / f_{p}$ is the wavelength of the $p$ th frequency step.

PSF of the MIMO array imaging system is the output image of the ideal point source for scanning position $\mathbf{r}=$ $(x, y)$, formulated by

$$
\begin{aligned}
& \operatorname{PSF}_{M}\left(\mathbf{r}, \mathbf{r}_{o}\right) \\
& =\sum_{m=0}^{M-1} \sum_{n=0}^{N-1} \sum_{p=0}^{P-1}\left(G\left(\mathbf{r}_{t, m}, \mathbf{r}_{r, n}, \mathbf{r}_{o}, f_{p}\right) G^{-1}\left(\mathbf{r}_{t, m}, \mathbf{r}_{r, n}, \mathbf{r}, f_{p}\right)\right) \\
& =\sum_{m=0}^{M-1} \sum_{n=0}^{N-1} \sum_{p=0}^{P-1} \frac{\left|\mathbf{r}_{t, m}-\mathbf{r}\right|\left|\mathbf{r}_{r, n}-\mathbf{r}\right|}{\left|\mathbf{r}_{t, m}-\mathbf{r}_{o}\right|\left|\mathbf{r}_{r, n}-\mathbf{r}_{o}\right|} \\
& \times e^{-j k_{p}\left(\left|\mathbf{r}_{t, m}-\mathbf{r}_{o}\right|+\left|\mathbf{r}_{r, n}-\mathbf{r}_{o}\right|-\left|\mathbf{r}_{t, m}-\mathbf{r}\right|-\left|\mathbf{r}_{r, n}-\mathbf{r}\right|\right)} .
\end{aligned}
$$

Equation (13) indicates that the PSF of the active imaging MIMO array is a spatial variant function of the target location $\mathbf{r}_{o}$, scanning position $\mathbf{r}$, and the frequency of the transmitted signal.

Similarly, we can get the approximate PSF from the equivalent virtual array, expressed as

$$
\begin{aligned}
& \operatorname{PSF}_{V}\left(\mathbf{r}, \mathbf{r}_{o}\right) \\
& =\sum_{i=0}^{M N-1} \sum_{p=0}^{P-1} \frac{\left|\mathbf{r}_{v, i}-\mathbf{r}\right|^{2}}{\left|\mathbf{r}_{v, i}-\mathbf{r}_{o}\right|^{2}} e^{-j 2 k_{p}\left(\left|\mathbf{r}_{v, i}-\mathbf{r}_{o}\right|-\left|\mathbf{r}_{v, i}-\mathbf{r}\right|\right)} .
\end{aligned}
$$

Actually, the concepts of PSF and beam pattern are identical under far-field narrow band condition. Let the carrier frequency of the narrow band signal is $f_{0}$, then $s(t)=e^{j 2 \pi f_{0} t}$. Under far-field condition, the following approximations hold for $m=0,1,2, \ldots, M-1$ and $n=0,1,2, \ldots, N-1$ :

$$
\begin{gathered}
\left|\mathbf{r}-\mathbf{r}_{t, m}\right|=|\mathbf{r}|-x_{t, m} \sin \theta \\
\left|\mathbf{r}_{o}-\mathbf{r}_{t, m}\right|=\left|\mathbf{r}_{o}\right|-x_{t, m} \sin \theta_{0} \\
\left|\mathbf{r}-\mathbf{r}_{r, n}\right|=|\mathbf{r}|-x_{r, n} \sin \theta \\
\left|\mathbf{r}_{o}-\mathbf{r}_{r, n}\right|=\left|\mathbf{r}_{o}\right|-x_{r, n} \sin \theta_{0} \\
\left|\mathbf{r}_{t, m}-\mathbf{r}\right| \approx\left|\mathbf{r}_{t, m}-\mathbf{r}_{o}\right| \\
\left|\mathbf{r}_{r, n}-\mathbf{r}\right| \approx\left|\mathbf{r}_{r, n}-\mathbf{r}_{o}\right|
\end{gathered}
$$

where $\theta$ and $\theta_{0}$ are the incidence angles of plane wave from $\mathbf{r}$ and $\mathbf{r}_{o}$, respectively.

Substitute (15a), (15b), (15c), (15d), (15e), and (15f) and $p=$ 0 into (13), the $\operatorname{PSF}_{M}\left(\mathbf{r}, \mathbf{r}_{o}\right)$ can be further expressed as the function of $\theta, \theta_{0}$, and $k_{0}$

$$
\begin{aligned}
& \operatorname{PSF}_{M}\left(\theta, \theta_{0}\right) \\
& =\sum_{m=0}^{M-1} \sum_{n=0}^{N-1} e^{j k_{0} 2\left(|\mathbf{r}|-\left|\mathbf{r}_{0}\right|\right)} e^{j k_{0}\left(x_{t, m}+x_{r, n}\right) \sin \theta_{0}} e^{-j k_{0}\left(x_{t, m}+x_{r, n}\right) \sin \theta}
\end{aligned}
$$

If we are only interested in the performance of the PSF over the azimuth direction $\theta$, the fixed coefficient $e^{j 2 k_{0}\left(|\mathbf{r}|-\left|\mathbf{r}_{o}\right|\right)}$ can be ignored. Using a change of variables

$$
\begin{aligned}
& w_{t, m}=e^{j k_{p} x_{t, m} \sin \theta_{0}}, \\
& w_{r, m}=e^{j k_{p} x_{r, n} \sin \theta_{0}} .
\end{aligned}
$$

Expression (16) would take the form

$$
\operatorname{PSF}_{M}\left(\theta, \theta_{0}\right)=\sum_{m=0}^{M-1} w_{t, m} e^{-j k_{p} x_{t, m} \sin \theta} \sum_{n=0}^{N-1} w_{r, n} e^{-j k_{p} x_{r, n} \sin \theta}
$$

which is the same expression as the radiation pattern of the MIMO array as shown in (4).

\section{UWB STVA Array}

4.1. UWB MIMO Array Design. Proper array design is helpful for simplifying the system structure and improving the imaging quality. The virtual aperture concept offers a basic framework for selecting transmit and receive aperture functions $[8,14]$. A virtual aperture desynthesis approach is applied to design the UWB MIMO array with uniform weighting according to the desired main lobe width and side lobe level in $[8,14]$. The essential step of the approach is the deconvolution process of (7). Unfortunately, given the two-way virtual aperture and the number of transmit and receive elements, the result of the de-convolution is not unique. Consider a linear virtual aperture with 32 virtual elements located uniformly within an aperture of $3.1 \mathrm{~m}$; five MIMO arrays with different topology are desynthesized each is composed of 2 transmit elements and 16 receive elements, as shown in Figure 2. Additional metric must be involved to evaluate the performance of the five different MIMO arrays. Further, in UWB MIMO array imaging application, the performance of a UWB MIMO array is related not only to the topology of the array and the number of the array elements, but also to the spectrum of the transmitted signal.

The spectrum of the transmitted signal may be approximated by its uniformly sampled discrete values, which is the form of stepped frequency waveform. In TWI application, stepped frequency continuous wave (SFCW) is one of the mostly used UWB waveforms $[5,20]$.

For UWB SFCW signal, the design process of an imaging MIMO array starts from the required resolution and the peak 


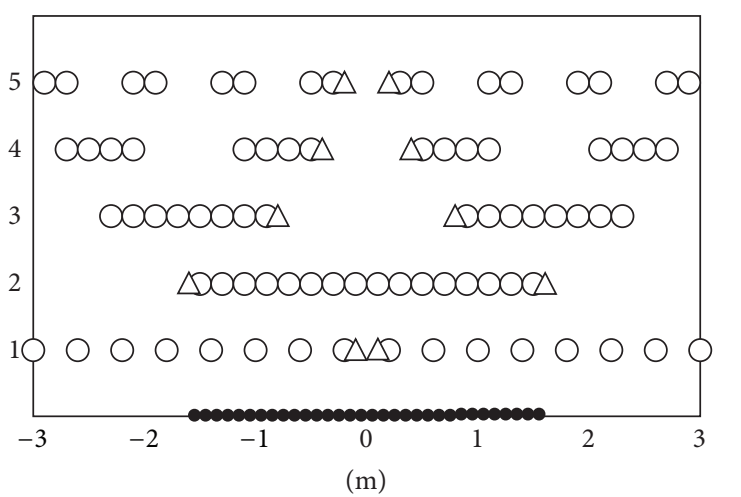

- Virtual element

$\triangle$ Transmit element

$\bigcirc$ Receive element

FIGURE 2: Linear MIMO arrays with the same virtual aperture array.

side lobe of the image, which determines the minimal aperture length and the number of virtual elements of the twoway virtual aperture from (14). In the far-field narrowband condition, the $3 \mathrm{~dB}$ width of the PSF main lobe in the azimuth direction steering to $\Theta$ from the boresight is approximately as [21]

$$
\Delta \theta \approx \frac{0.886 \lambda}{2 L \cos (\Theta)},
$$

where $L$ is the two-way aperture length and $\lambda$ is the wavelength of the transmitted waveform.

Then the number of transmit and receive elements can be chosen under the relation

$$
N_{v}=N_{t} \cdot N_{r},
$$

where $N_{t}, N_{r}$, and $N_{v}$ are the numbers of transmit, receive, and the virtual elements, respectively.

In the next step, the transmit and receive array topology can be derived through deconvolution of (7), which is similar to the process in $[5,20]$. The de-convolution procedure is not unique and allows for multiple solutions. To select the optimal one among them, the monostatic-to-bistatic equivalence condition in radar signal processing should be considered. Remarking that the radar cross section (RCS) of a point scatterer (the object is modeled as a set of discrete scattering centers) is dependent on the incidence and reflection angle, the equivalence is valid only at a small range of angle. Thus, we hope to decrease the overall length of the physical aperture including all the transmit and receive elements, and we also hope that the transmit aperture and the receive aperture have similar illumination geometry to all the point scatterers. For the case listed in Figure 2, option 2 with the two transmit elements located at either end of the linear uniform receive array is the optimal selection. We called the linear MIMO with similar topology as the split transmitter virtual aperture (STVA) array.

After that, the configuration of the STVA array is adjusted considering the mutual coupling and the UWB antenna

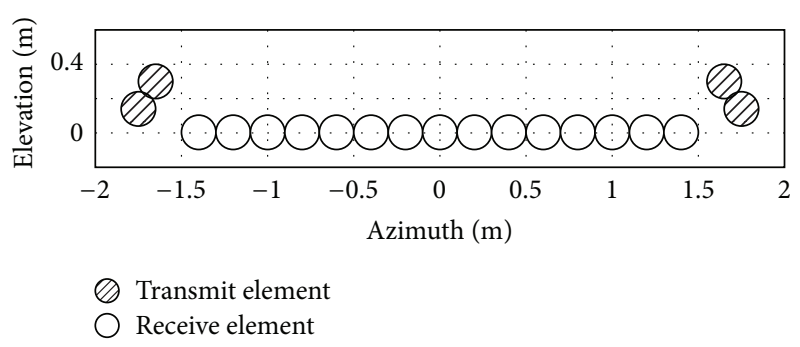

(a)

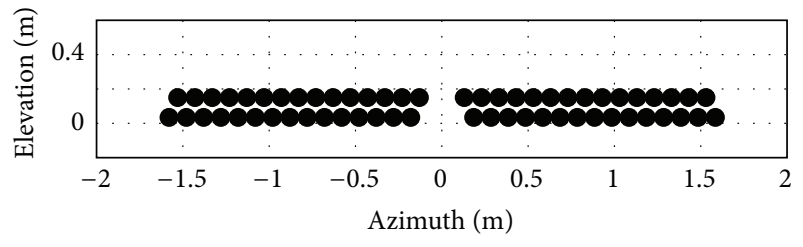

- Virtual element

(b)

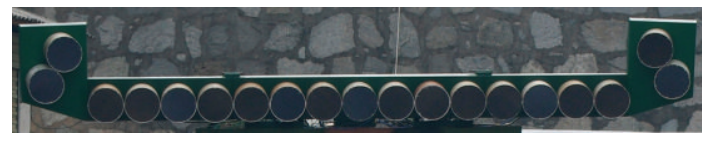

(c)

FIGURE 3: Designed split transmitter virtual aperture array. (a) Topology; (b) equivalent two-way virtual aperture; (c) front view of the of the STVA array.

element size. For array transmitting continuous waveform, the direct coupling from the transmit to the receive elements decreases the receiver sensitivity, saturates the receiver, and even damages the receiver. The transmit and receive elements should be placed at a proper distance to improve the T/R isolation, assuring that the receiver is not jammed. The transmit elements may move a little distance away at both azimuth and elevation levels. It should be noted that theoretically the displacement in elevation of the transmit elements does not influence the array performance in the azimuth direction.

Finally, during the array design, the center carrier frequency is used to calculate the main lobe width and side lobe level. In TWI application, the transmitted UWB signal extends across multiple octaves in the frequency domain. The spacing of the designed array elements may cause grating lobes at the higher operational frequency. If this does occur, the number of transmit elements should be increased.

4.2. UWB STVA Array Analysis. Using the array design approach described above, an example of STVA array has been designed for TWI application. The topology of the STVA array is shown in Figure 3(a). The STVA array consists of 4 transmit and 15 receive elements. The receive array consists of 15 elements with interelement spacing of $0.2 \mathrm{~m}$. The four transmit elements are located at $(-1.75 \mathrm{~m}, 0.1268 \mathrm{~m})$, $(-1.65 \mathrm{~m}, 0.3 \mathrm{~m}),(1.65 \mathrm{~m}, 0.3 \mathrm{~m})$, and $(1.75 \mathrm{~m}, 0.1268 \mathrm{~m})$, respectively, two at either end of the receive array. The physical aperture length of the array is $3.5 \mathrm{~m}$ (the largest distance between two elements of the array measured from 


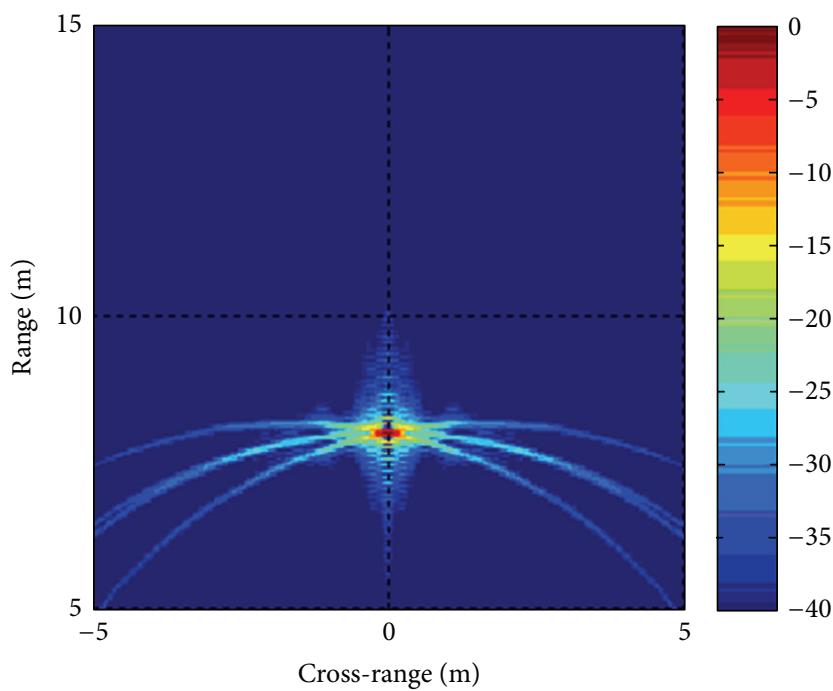

(a)

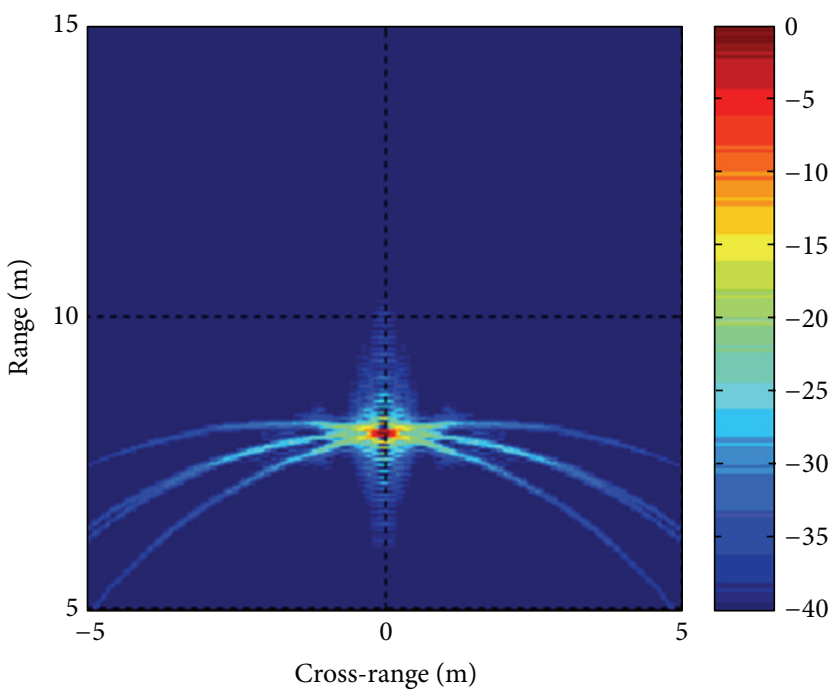

(b)

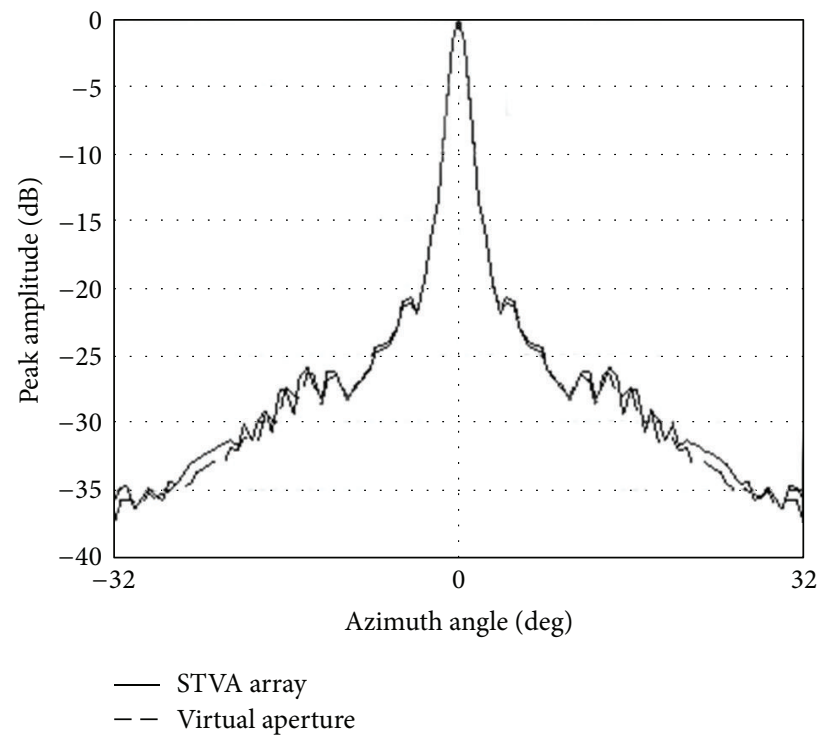

(c)

FIGURE 4: Comparison of the PSFs between the STVA array and its virtual array with focal point at $8 \mathrm{~m}$. (a) STVA array. (b) Virtual array. (c) Peak amplitude pattern in azimuth angle.

phase center to phase center). The transmit elements are located at least $0.37 \mathrm{~m}$ away from the nearest receive element (from phase center to phase center) to reduce the direct coupling from the transmit elements to the receive elements to an acceptable level. Using the concept of virtual phase center, a virtual aperture with 60 virtual transmit/receive (T/R) elements is synthesized, as shown in Figure 3(b). The interelement spacing of the synthesized virtual aperture is $0.05 \mathrm{~m}$ except a gap of $0.25 \mathrm{~m}$ at the midpoint, synthesizing a total aperture length of $3.15 \mathrm{~m}$. The gap at the midpoint is due to the separation of the transmit elements. And it will result in a slight acceptable increase of the side lobe level, which will be shown in the following. Both the transmit and receive antennas are UWB Archimedean spirals with a diameter of
$0.2 \mathrm{~m}$, working at the frequency band of $0.5 \mathrm{G} \mathrm{Hz} \sim 2.0 \mathrm{GHz}$. The array is mounted on the top of a van with the front view of the STVA array shown in Figure 3(c).

It has been demonstrated that under the narrowband far-field condition, the equivalence of the PSF between the MIMO array and its virtual aperture is exact, and the virtual aperture fully describes the overall performance of the original MIMO array, while under wideband conditions, the equivalence becomes approximate. Therefore, it is necessary to compare the PSF between the original MIMO array and its virtual aperture to verify the feasibility of the design approach.

For narrow band array, the boundary of the far-field region is $R_{f}>L^{2} / \lambda$, where $L$ is the largest distance 


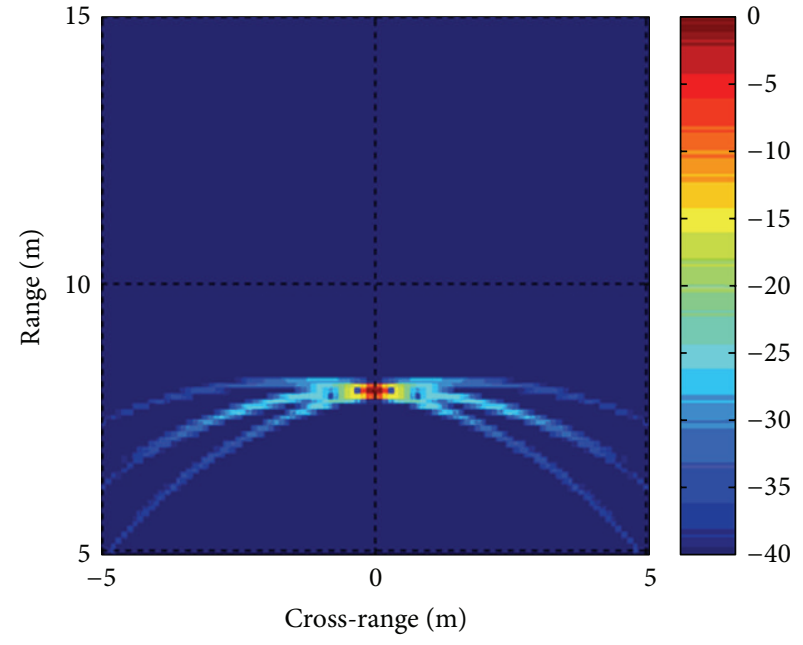

(a)

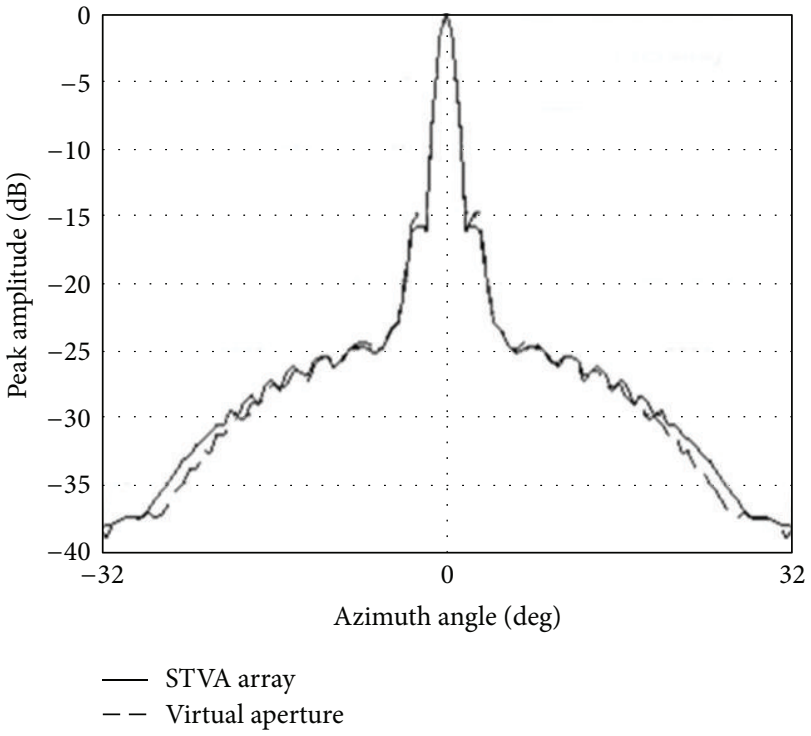

(b)

FIGURE 5: Imaging results of the STVA array with Hamming function in frequency. (a) Imaging result. (b) Peak amplitude pattern in azimuth angle.

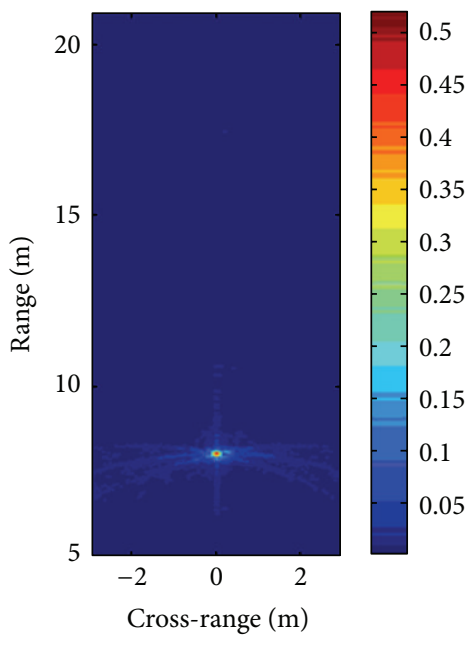

(a)

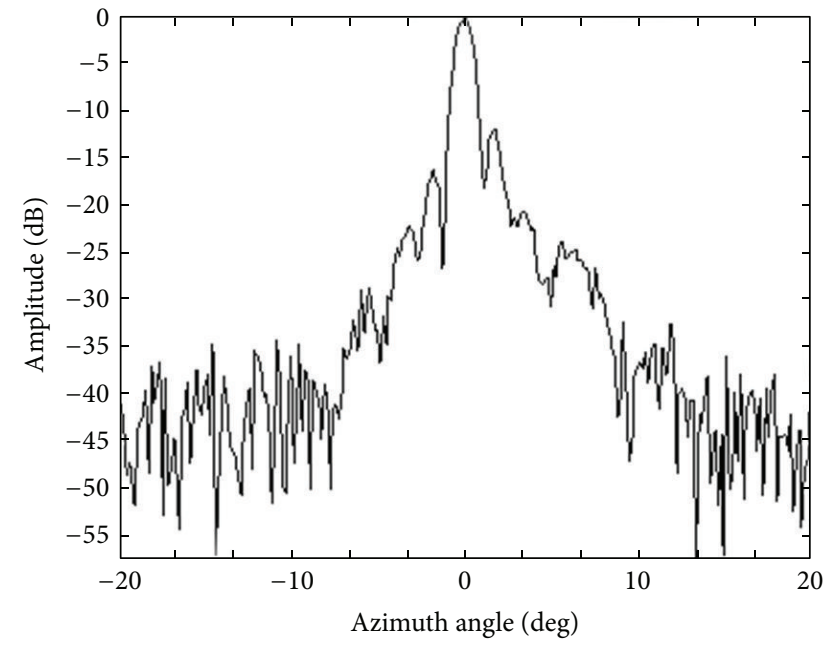

(b)

FIGURE 6: Image of metal trihedral corner reflector. (a) Imaging result. (b) Peak amplitude pattern in azimuth angle.

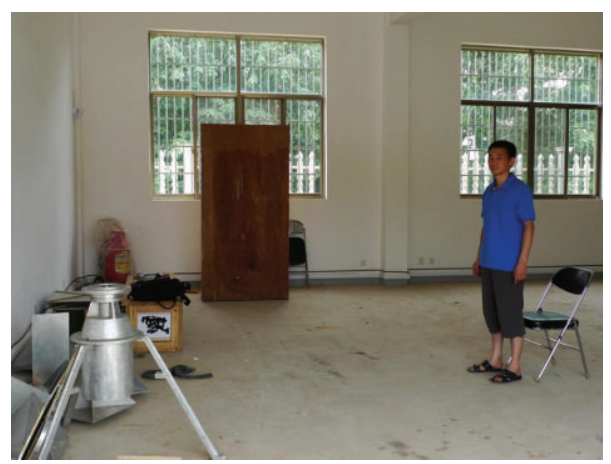

FIGURE 7: Experimental imaging scene. 


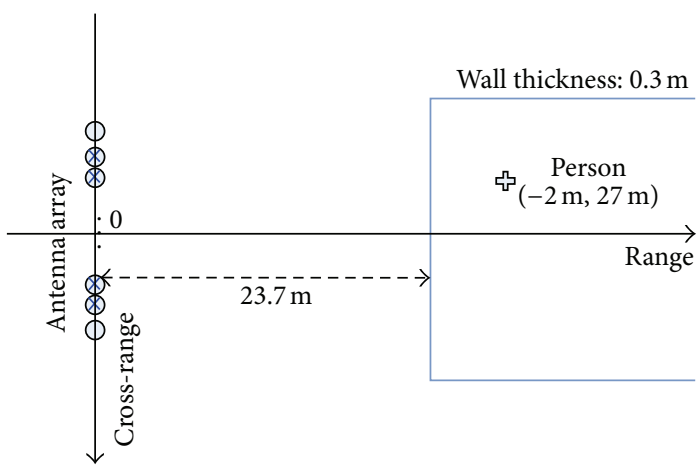

(a)

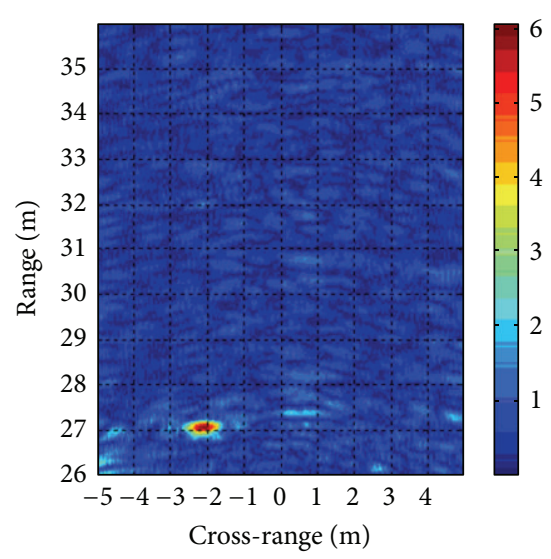

(b)

FIGURE 8: Image of a person inside the building. (a) Geometry of imaging. (b) Imaging result.

between two elements of the array. Using this equation, for the designed UWB STVA array, we get the far-field boundary of $16.5 \mathrm{~m}$ at the lowest operational frequency and $66 \mathrm{~m}$ at the highest operational frequency. For a vehicle-borne TWI system, the detection range is usually between $5 \mathrm{~m}$ and $50 \mathrm{~m}$, which spans the near-and far-field boundary region. Thus, the influence of target distance on the performance of the MIMO array should also be investigated.

The PSF of the original STVA array and the PSF of its virtual aperture with focal point at the location $(0,8 \mathrm{~m})$ are illustrated in Figures 4(a) and 4(b), respectively. The peak amplitude patterns in the azimuth angle of both the PSFs are shown in Figure 4(c) for more direct comparison, which demonstrated that both patterns are almost equivalent. The farther the focal distance, the smaller the approximation error.

The cross-range resolution of the point target at $8 \mathrm{~m}$ is about $0.246 \mathrm{~m}$. For the large relative bandwidth of the transmitting UWB waveform (120\%), the first side lobe is about $22 \mathrm{~dB}$ lower than the peak of the main lobe. In theory, the wider the bandwidth, the lower the side lobe. In the ideal narrow band case, the first side lobe level is about $-13 \mathrm{~dB}$.

During the calculation of PSFs, frequency weight coefficients are set to unity, which will result in the high level range side lobe of about $-13 \mathrm{~dB}$. In practical imaging processing, different types of frequency weighting function are used to reduce the range side lobe level. Unfortunately, frequency weighting function also affects the performance of the azimuth of the UWB array. The image results of the same ideal point target with hamming weighting function in frequency are shown in Figure 5. The range side lobe level is reduced to about $-40 \mathrm{~dB}$, while the azimuth side lobe level is increased to $-15 \mathrm{~dB}$.

The simulation results are verified with measured data. A $0.1 \mathrm{~m}$ metal trihedral corner reflector was placed $8 \mathrm{~m}$ in front of the STVA array on a plane ground. The resulting image is shown in Figure 6. The cross-range resolution and the location distribution of the side lobe are much the same as the simulation, but the level of the first right side lobe is a little higher than the simulation, which is likely due to the nonideality of the measurement setup.

\section{Experimental Results}

The designed STVA array has been successfully applied in TWI application. One of the active imaging scenes is shown in Figure 7. The imaging array is placed at the left side of the cinderblock garage, and the array is parallel to the side wall, at a distance of $23.7 \mathrm{~m}$. The thickness of the cinderblock wall is about $30 \mathrm{~cm}$. The garage is $10 \mathrm{~m}$ wide and $16 \mathrm{~m}$ deep. During the acquisition of data, a man with the height of $172 \mathrm{~cm}$ walks between the front wall and the back wall along the range direction in the room.

A standard differential back projection (BP) imaging algorithm [5] is adopted to process the acquired data. The time interval of differential processing is $300 \mathrm{~ms}$. The static background clutter is eliminated and the moving person is imaged. A series of differential images is acquired, and walking history of the person is clearly shown combining all the frames of images. One of the images with the person located at $(-2 \mathrm{~m}, 27 \mathrm{~m})$ is shown in Figure 8.

\section{Conclusion}

The virtual aperture concept offers a basic framework for selecting transmit and receive aperture functions of MIMO array under narrow band far-field condition. In this paper, the MIMO array design approach is extended for the near-field wide band case. Combining the approach of virtual aperture decomposition with the monostatic-to-bistatic equivalence theorem, a kind of linear UWB MIMO array with the shortest physical aperture length among these synthesizing the same virtual aperture, the STVA array, was designed for through the wall imaging. The PSF of the original STVA array and the PSF of its virtual aperture are compared, demonstrating that the approximation error is negligible even at the nearest focal range. 
The imaging performance of the designed STVA array in the near field is fully analyzed through both numerical and measured data. The designed STVA array has been successfully applied to imaging moving targets inside buildings.

\section{References}

[1] G. L. Charvat, L. C. Kempel, E. J. Rothwell, C. M. Coleman, and E. L. Mokole, "An ultrawideband (UWB) switched-antennaarray radar imaging system," in Proceedings of the 4th IEEE International Symposium on Phased Array Systems and Technology (Array '10), pp. 543-550, October 2010.

[2] A. G. Yarovoy, T. G. Savelyev, P. J. Aubry, P. E. Lys, and L. P. Ligthart, "UWB array-based sensor for near-field imaging," IEEE Transactions on Microwave Theory and Techniques, vol. 55, no. 6, pp. 1288-1295, 2007.

[3] A. Nelander, "Switched array concepts for 3-D radar imaging," in Proceedings of the IEEE International Radar Conference (RADAR '10), pp. 1019-1024, Washington, DC, USA, May 2010.

[4] K. E. Browne, R. J. Burkholder, and J. L. Volakis, “Throughwall opportunistic sensing system utilizing a low-cost flat-panel array," IEEE Transactions on Antennas and Propagation, vol. 59, no. 3, pp. 859-868, 2011.

[5] B. Lu, Q. Song, Z. Zhou, and X. Zhang, "Detection of human beings in motion behind the wall using SAR interferogram," IEEE Geoscience and Remote Sensing Letters, vol. 9, no. 5, pp. 968-971, 2012.

[6] F. Ahmad, M. G. Amin, and S. A. Kassam, "Synthetic aperture beamformer for imaging through a dielectric wall," IEEE Transactions on Aerospace and Electronic Systems, vol. 41, no. 1, pp. 271-283, 2005.

[7] F. Ahmad, Y. Zhang, and M. G. Amin, “Three-dimensional wideband beamforming for imaging through a single wall," IEEE Geoscience and Remote Sensing Letters, vol. 5, no. 2, pp. 176-179, 2008.

[8] X. Zhuge and A. Yarovoy, "Near-field ultra-wideband imaging with two-dimensional sparse MIMO array," in Proceedings of the 4th European Conference on Antennas and Propagation (EuCAP '10), pp. 1-4, Barcelona, Spain, April 2010.

[9] X. Zhuge and A. G. Yarovoy, "A sparse aperture MIMO-SARbased UWB imaging system for concealed weapon detection," IEEE Transactions on Geoscience and Remote Sensing, vol. 49, no. 1, pp. 509-518, 2011.

[10] D. W. Bliss and K. W. Forsythe, "Multiple-input multipleoutput (MIMO) radar and imaging: degrees of freedom and resolution," IEEE Transactions on Ultrasonics, Ferroelectrics and Frequency Control, vol. 43, no. 1, pp. 7-14, 1996.

[11] J. Li and P. Stoica, "MIMO radar with colocated antennas," IEEE Signal Processing Magazine, vol. 24, no. 5, pp. 106-114, 2007.

[12] F. Ahmad and S. A. Kassam, "Coarray analysis of the wideband point spread function for active array imaging," Signal Processing, vol. 81, no. 1, pp. 99-115, 2001.

[13] F. Ahmad, G. J. Frazer, S. A. Kassam, and M. G. Amin, "Design and implementation of near-field, wideband synthetic aperture beamformers," IEEE Transactions on Aerospace and Electronic Systems, vol. 40, no. 1, pp. 206-220, 2004.

[14] Z. Li, T. Jin, B. Chen, and Z. Zhou, "A coarray based MIMO array design method for UWB imaging," in Proceedings of the IET International Conference on Radar Systems, pp. 1-4, Glasgow, UK, October 2012.
[15] W.-Q. Wang, "Virtual antenna array analysis for MIMO synthetic aperture radars," International Journal of Antennas and Propagation, vol. 2012, Article ID 587276, 10 pages, 2012.

[16] R. T. Hoctor and S. A. Kassam, "The unifying role of the coarray in aperture synthesis for coherent and incoherent imaging," Proceedings of the IEEE, vol. 78, no. 4, pp. 735-752, 1990.

[17] J. L. Schwartz and B. D. Steinberg, "Ultrasparse, ultrawideband arrays," IEEE Transactions on Ultrasonics, Ferroelectrics, and Frequency Control, vol. 45, no. 2, pp. 376-393, 1998.

[18] V. Murino, A. Trucco, and A. Tesei, "Beam pattern formulation and analysis for wide-band beamforming systems using sparse arrays," Signal Processing, vol. 56, no. 2, pp. 177-183, 1997.

[19] C. J. Bradley, P. J. Collins, D. G. Falconer, J. Fortuny-Guasch, and A. J. Terzuoli Jr., "Evaluation of a near-field monostatic-tobistatic equivalence theorem," IEEE Transactions on Geoscience and Remote Sensing, vol. 46, no. 2, pp. 449-456, 2008.

[20] M. G. Amin, Through-the-Wall Radar Imaging, CRC Press, New York, NY, USA, 2011.

[21] G. Franceschetti and R. Lanari, Synthetic Aperture Radar Processing, CRC Press, Boca Raton, Fla, USA, 1999. 

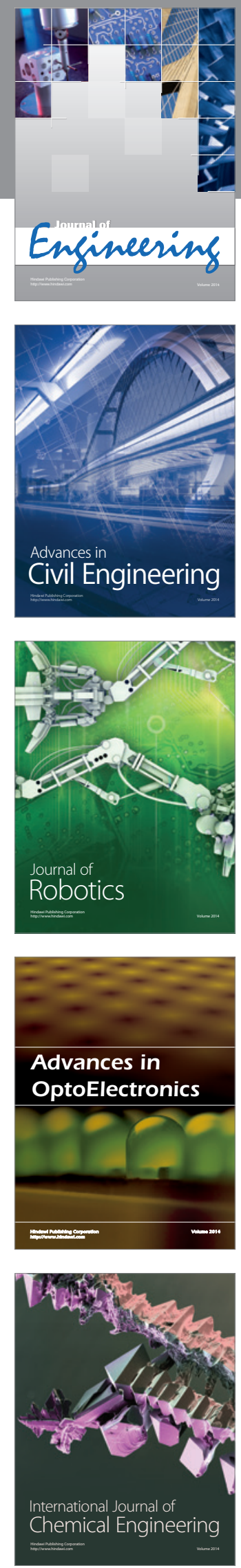

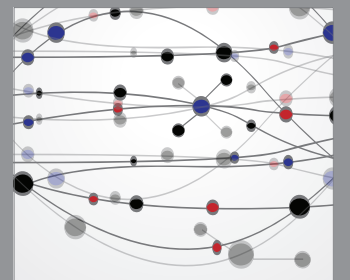

The Scientific World Journal
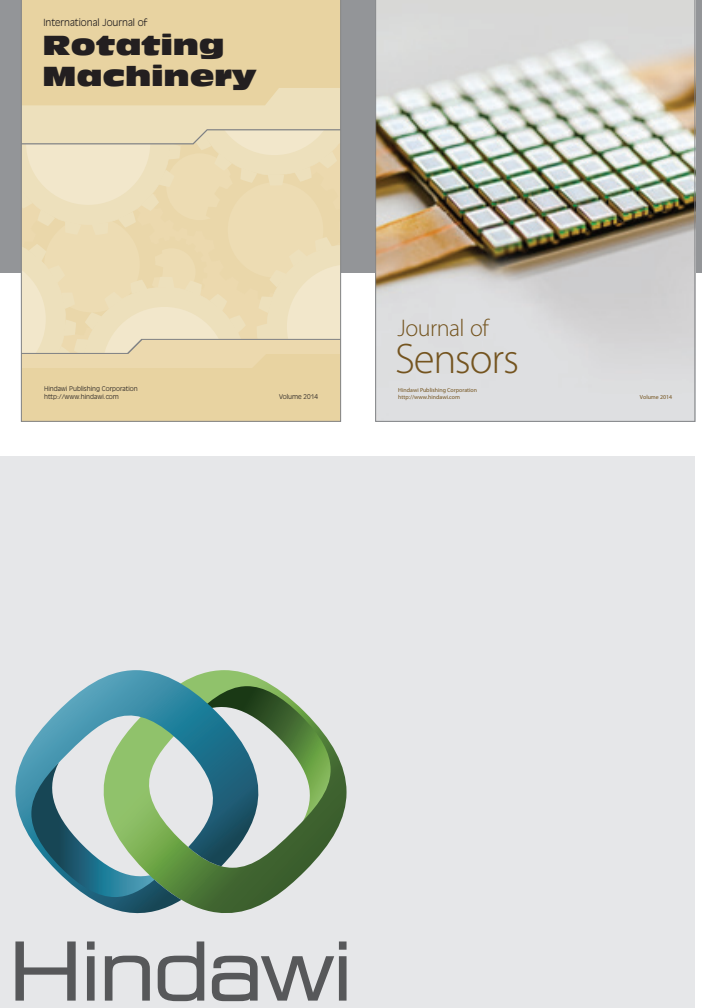

Submit your manuscripts at http://www.hindawi.com
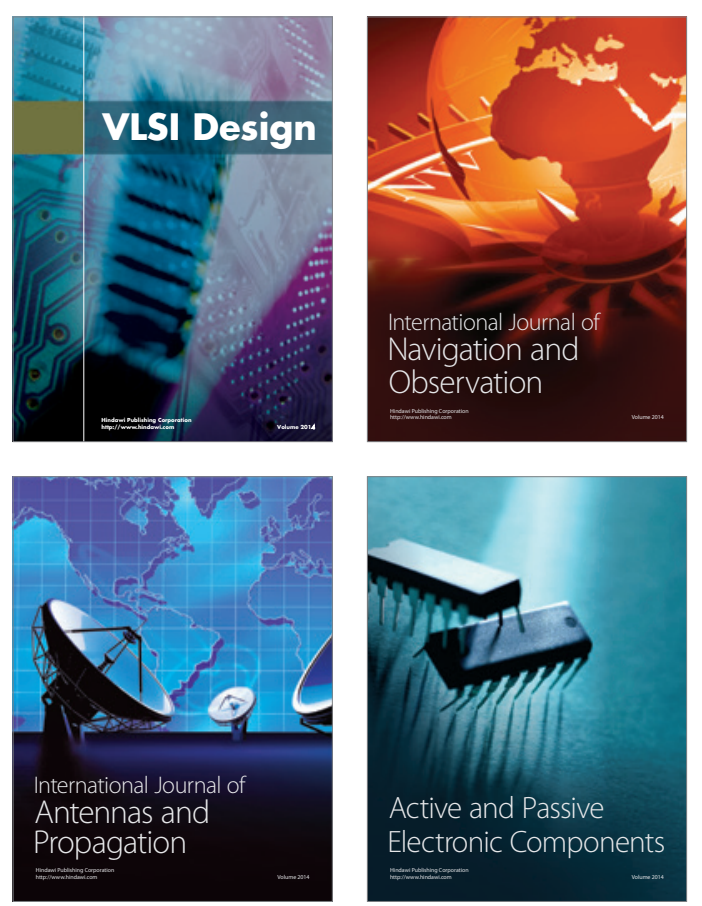
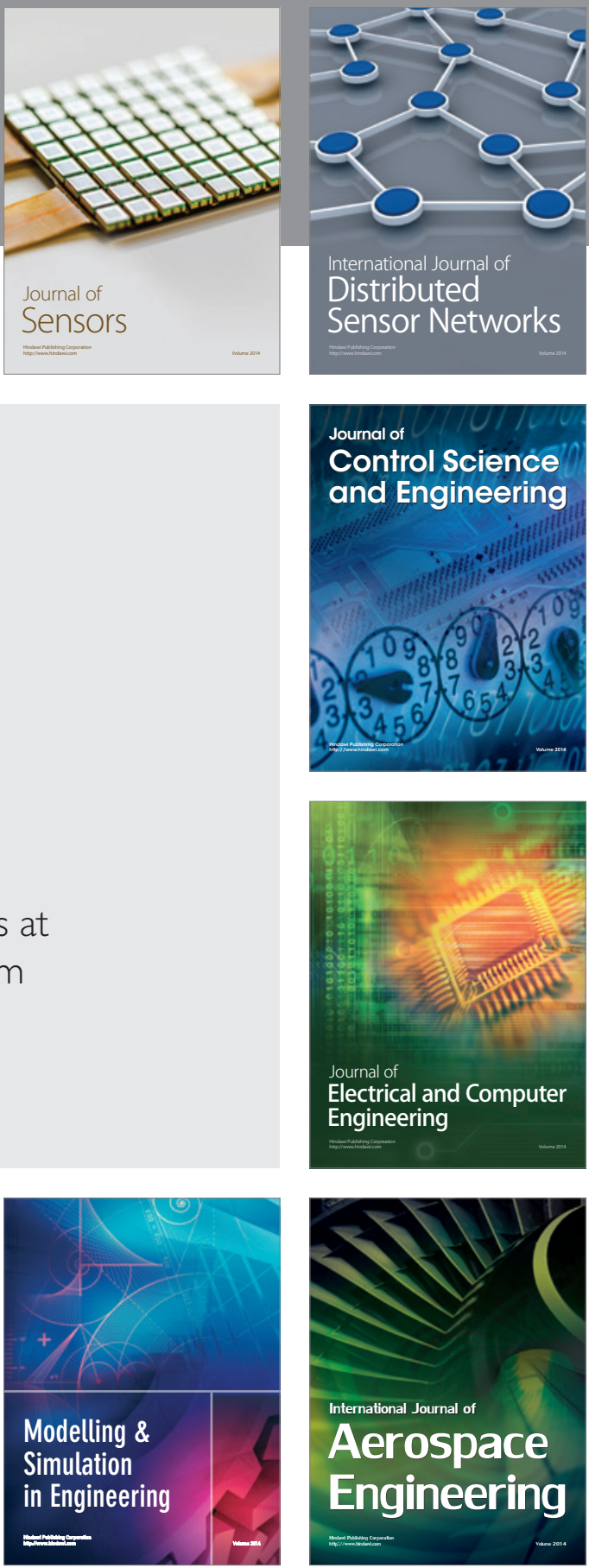

Journal of

Control Science

and Engineering
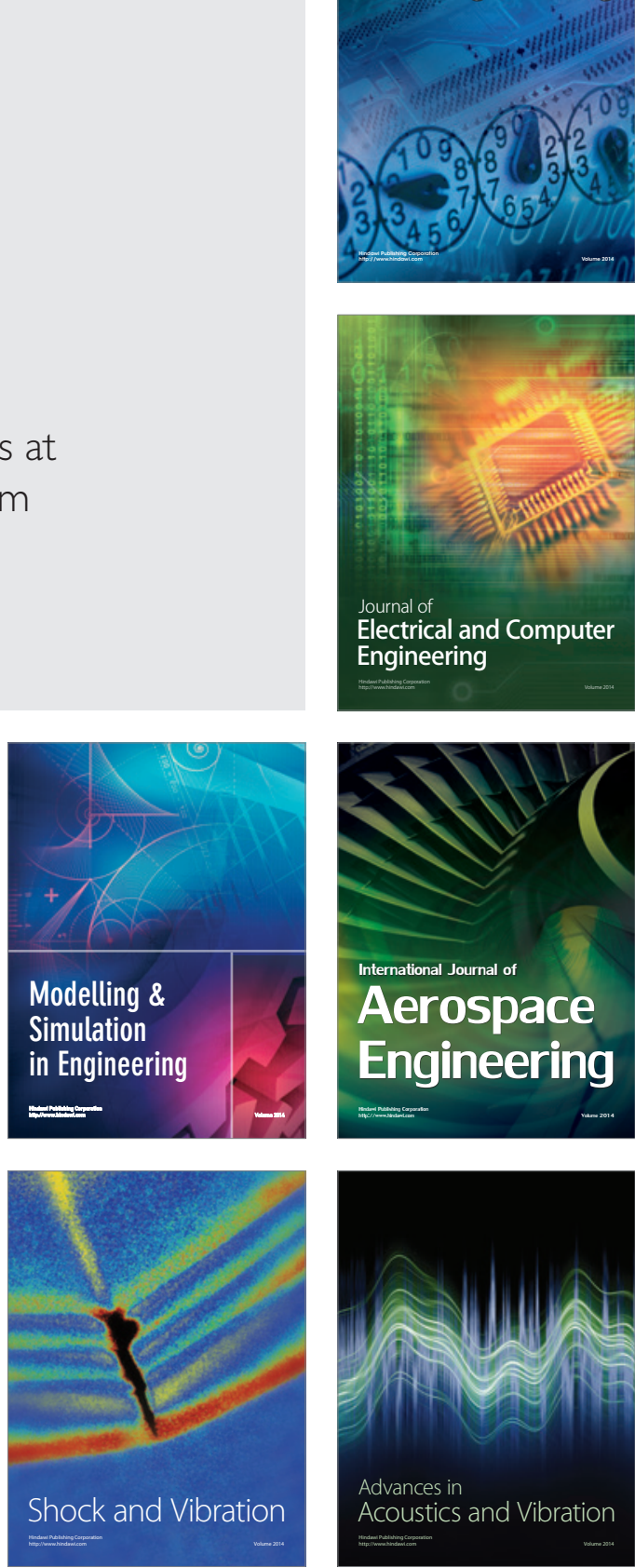